5. World Health Organization (2015). Guidelines for the Prevention, Care and Treatment of Persons with Chronic Hepatitis B Infection. Pp. 1-166.

6. Sarin, S. K., Kumar, M., Lau, et al (2016). Asian-Pacific clinical practice guidelines on the management of hepatitis B: a 2015 update. Hepatology international, 10(1), 1-98. https://doi.org/10.1007/s12072-015-9675-4

7. Nagueh SF, Smiseth OA, Appleton CP, et al. Recommendations for the Evaluation of Left
Ventricular Diastolic Function by Echocardiography: An Update from the American Society of Echocardiography and the European Association of Cardiovascular Imaging. J Am Soc Echocardiogr. 2016; 29:277-314.

8. Yuan W., Lu, H. Z., Mei, X., et al (2019). Cardiac health in patients with hepatitis B virusrelated cirrhosis. Medicine; 98(13), e14961. https://doi.org/10.1097/MD.0000000000014961

\title{
HIÊUU QUẢ PHỐI HỢP KHÁNG SINH IN VITRO TRÊN VI KHUẨN GRAM ÂM KHÁNG CARBAPENEM
}

\begin{abstract}
TÓM TẮT.
Đă̆t vấn đề: Theo Tổ chức $Y$ tế thế giới (WHO), vi khuẩn (VK) Acinetobacter baumannii, Pseudomonas aeruginosa, Klebsiella pneumoniae kháng carbapenem (APK-CR) là những vi khuẩn (VK) có mức cảnh báo cao nhất, cần ưu tiên phát triển các loại kháng sinh (KS) mới do tình trạng kháng thuốc đáng báo động [7]. Mục tiêu: Khảo sát MIC và hiệu quả phối hợp KS in vitro của meropenem (ME) - colistin (COL) và meropenem - ciprofloxacin (CIP) trên các chủng VK APK-CR. Phương pháp: Nghiên cứu mô tả cắt ngang. Các chủng APK-CR được phân lập tai Bênh viện Đại học Y Dược TP. HCM từ tháng 12/2020 đến tháng 06/2021. Kết quả: Có 151 chủng gồm 51 chủng $A$. baumannii, 50 chủng $P$. aeruginosa và 50 chủng $K$. pneumoniae. MIC của ME và CIP trên các chủng APKCR đều cao (chiếm 92-100\%); có $6 \%$ chủng $P$. aeruginosa và $10 \%$ chủng $\mathrm{K}$. pneumoniae là có MIC kháng COL. Hiệu quả hiệp đồng và cộng hợp trong phối hợp KS in vitro của ME-COL trên APK-CR có tỷ lệ lần lượt là $58,8 \%$ và $41,2 \%, 32 \%$ và $60 \%, 20 \%$ và $60 \%$. Hiệu quả hiệp đồng và cộng hợp trong phối hợp $\mathrm{KS}$ in vitro của ME-CIP trên APK-CR có tỷ lệ lần lượt là $33,3 \%$ và $45,1 \%, 30 \%$ và $60 \%, 42 \%$ và $44 \%$. Kết luâan: $A P K-C R$ đề kháng với $M E$, CIP với tỷ lê rất cao. Phối hợp ME-COL và ME-CIP trên APK-CR có kết quả hiệp đồng và cộng hợp làm giảm tỷ lệ đề kháng KS của APK-CR.
\end{abstract}

Từ khoá: phối hợp kháng sinh, APK-CR (A. baumannii - P.aeruginosa - K.pneumoniae kháng carbapenem).

\section{SUMMARY}

\section{IN-VITRO EFFICACY OF ANTIBIOTIC}

${ }^{1}$ Trường Đại học Y Khoa Phạm Ngọc Thạch

${ }^{2} Đ a ̣ i$ học Y Dước TPHCM

${ }^{3}$ Bềnh viên Đai hoc Y Dước TPHCM

Chịu trách nhiệm chính: Ngô Hoàng Ngọc Thanh

Email: hoang.nthanh71@gmail.com

Ngày nhận bài: 16.10 .2021

Ngày phản biên khoa hoc: 11.10.2021

Ngày duyệt bài: 18.10.2021
Ngô Hoàng Ngọc Thanh ${ }^{1}$, Phạm Thái Bình²,
Cao Minh Nga

\section{COMBINATION ON THE CARBAPENEM-}

RESISTANT GRAM-NEGATIVE BACTERIA

Background: According to the World Health Organization, Acinetobacter baumannii, Pseudomonas aeruginosa, and Klebsiella pneumoniae carbapenem resistance (APK-CR) are the most dangerous bacteria. The new antibiotic development should be prioritized for APK-CR because of their antibiotic resistance seriously. Objective: Evaluate the resistance of APK and the effectiveness of in vitro antibiotic combination of meropenem (ME) - colistin (COL) and meropenem ciprofloxacin (CIP) to these bacteria strains. Methods: Descriptive and analytical cross sectional study. APK-CR strains were collected at University Medical Center in Ho Chi Minh City from December 2020 to June 2021. Results: There were 151 APK-CR strains (51 A. baumannii, $50 \mathrm{P}$. aeruginosa and $50 \mathrm{~K}$. pneumoniae). The MICs of ME and CIP on APK-CR strains were both high (accounting for $92-100 \%$ ); $6 \%$ of $\mathrm{P}$. aeruginosa and $10 \%$ of $\mathrm{K}$. pneumoniae were MIC resistant to $\mathrm{COL}$. The combination of ME-COL gave high synergistic and additive effectiveness on $A$. baumannii at the rate of $58.8 \%$ and $41.2 \%$, while $P$. aeruginosa showed the lower rate of $32 \%$ and $60 \%$, K. pneumoniae was at the rate of $20 \%$ and $60 \%$. The combination of ME-CIP gave high synergistic and additive effectiveness on $\mathrm{K}$. pneumoniae at the rate of $42 \%$ and $44 \%$, while A. baumannii showed the lower rate of $33.3 \%$ and $45.1 \%$, P. aeruginosa was at the rate of $30 \%$ and $60 \%$. Conclusion: APK-CR resists to $\mathrm{ME}$ and CIP with a very high rate. The antibiotic combination in vitro of ME-COL and ME-CIP on APK-CR has the synergistic and additive effectiveness.

Key words: combination antibiotic, APK-CR (A. baumannii - P. aeruginosa - K. pneumoniae carbapenem resistance).

\section{I. ĐĂTT VẤN ĐỀ}

Theo Cơ quan Quản lý Dược phẩm Châu Âu, mỗi năm có 33.000 ca tử vong do nhiễm VK đa kháng thuốc và chi phí lên đến 1,5 tỷ Euro mỗi năm [5]. WHO đưa ra danh sách các VK cần ưu tiên phát triển KS mới do tình trạng kháng thuốc 
đáng báo động, trong đó APK-CR có mức cảnh báo cao nhất [7].

Tại Bệnh viện Đại học Y Dược TP.HCM, tỷ lệ VK kháng carbapenem có chiêu hướng tăng mạnh trong vòng một thập kỷ qua, cụ thể trên Acinetobacter spp. tăng từ $39 \%$ lên $90 \%$, Pseudomonas spp. tăng từ $3 \%$ lên $64 \%$ và Klebsiella spp. tăng từ 4\% lên 45\% [1] [2] [3]. Hơn thễ nữa, $A$. baumannii có tỷ lệ đề kháng cao và là VK được định danh chủ yếu trong các loài Acinetobacter spp. [4], bên cạnh đó $P$. aeruginosa và $K$. pneumoniae cũng là VK được định danh và gây bệnh chủ yếu trong loài Pseudomonas spp. và Klebsiella spp. [1] [2] [3].

Việc tiến hành nghiên cứu nhằm mục tiêu: Xác định MIC của ME, COL, CIP trên APK-CR; Khảo sát hiệu quả phối hợp ME-COL và ME-CIP trên APK-CR bằng phương pháp vi pha loãng và so sánh khi sử dụng từng loại KS đơn lẻ.

\section{II. ĐỐI TƯợNG VÀ PHƯƠNG PHÁP NGHIÊN CỨU}

2.1. Đối tượng. Có 151 chủng APK gồm 51 chủng $A$. baumannii, 50 chủng $P$. aeruginosa và 50 chủng $\mathrm{K}$. pneumoniae thỏa tiêu chuẩn lựa chọn tại Bệnh viện Đại học Y Dược TP. HCM từ tháng 12/2020 đến tháng 6/2021 từ các mẫu bệnh phẩm (đàm, mủ, dịch, nước tiểu) cho kết quả kháng sinh đồ kháng ít nhất một trong bốn KS imipenem, meropenem, ertapenem, doripenem. Mẫu đàm: đạt $\geq 25$ bạch cầu và $\leq 10$ tế bào biểu mô/ phết nhuộm Gram. Mẫu nước tiểu: số lượng VK cùng loài $\geq 10^{5} \mathrm{CFU} / \mathrm{ml}$.

\subsection{Phương pháp nghiên cứu}

Thiết kế nghiên cứu: mô tả cắt ngang.

Tiến hành: Định danh VK bằng kit định danh Panel BD Phoenix ${ }^{\mathrm{TM}}$ NID, chạy trên máy $\mathrm{BD}$ Phoenix ${ }^{T M}$ M50 (Becton Dickinson). Phân loại nhạy (S), trung gian (I), kháng (R) với KS dựa theo tiêu chuẩn CLSI 2020. Khảo sát hiệu quả phối hợp KS bằng phương pháp vi pha loãng trên khay 96 giếng và tính chỉ số FIC như sau:

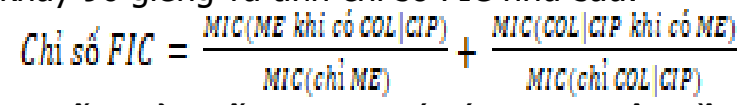

Kết quả phối hợp KS có tác dụng hiệp đồng khi FIC $\leq 0,5$, cộng hợp khi FIC $>0,5-1$, độc lập khi FIC $>1-<4$ hoặc đối kháng khi FIC $\geq 4$. So sánh các tỷ lệ bằng các test thống kê.

Y đức: được thông qua tại Hội đồng Đạo đức trong nghiên cứu y sinh học tại Đại học Y Dược TP.HCM số 916/ HĐĐĐ-ĐHYD ngày 30/11/2020.

\section{KẾT QUẢ NGHIÊN CỨU VÀ BÀN LUẬN 3.1. MIC kháng sinh ME, COL, CIP}

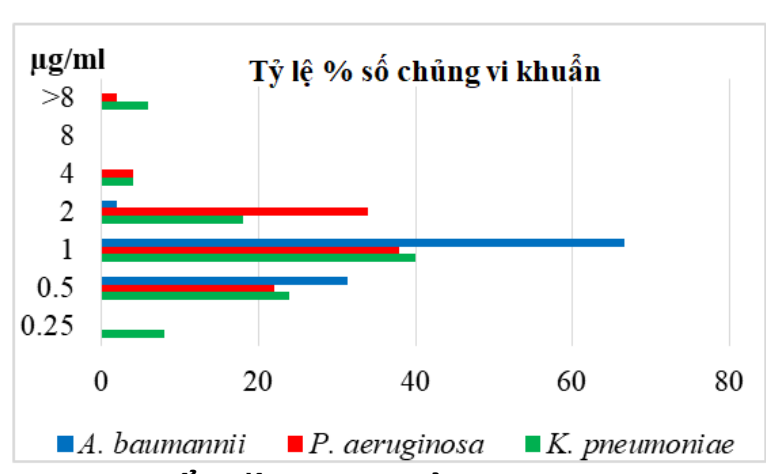

Biểu đồ 1: MIC của KS colistin

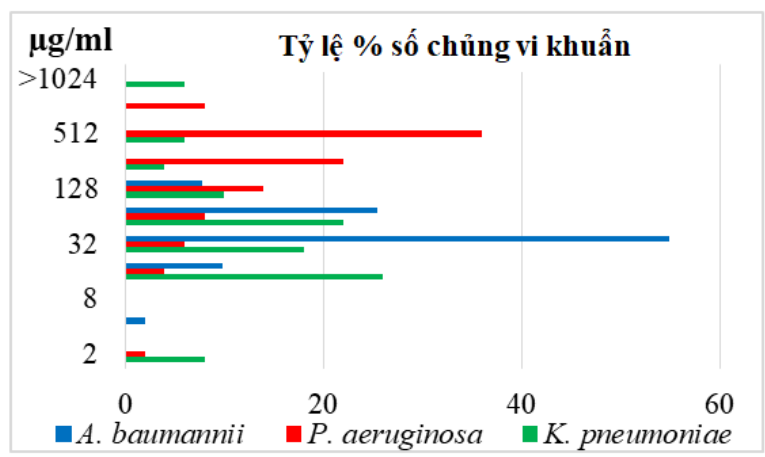

\section{Biểu đồ 2: MIC của KS meropenem}

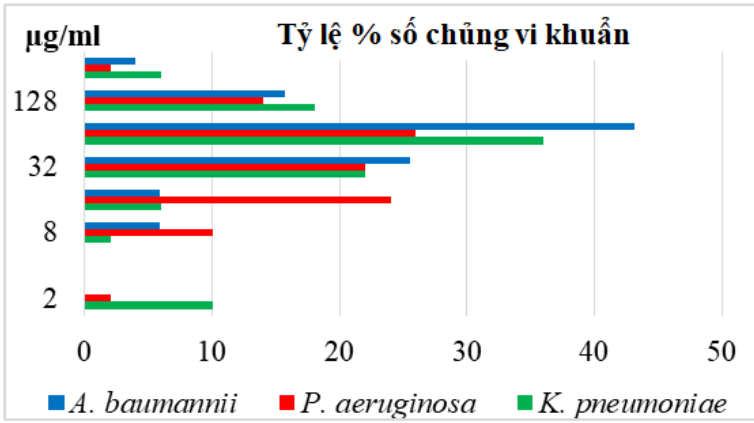

Biểu đồ 3: MIC của KS ciprofloxacin

Dựa vào Biểu đồ 1 , Biểu đồ 2 và Biểu đồ 3 : Khi so với MIC $\geq 8 \mu \mathrm{g} / \mathrm{ml}$ để xác định VK kháng với ME [6] thì mức độ đề kháng KS của $A$. baumannii không quá cao, còn trên $P$. aeruginosa thì mức độ đề kháng $\mathrm{KS}$ rất cao, cao gấp 4 lần $A$. baumannii (MIC $\geq 32 \mu \mathrm{g} / \mathrm{ml}$ chiếm $79 \%$ so với MIC $\geq 128 \mu \mathrm{g} / \mathrm{ml}$ trên $80 \%$ ). Khi so với MIC $\geq 4 \mu \mathrm{g} / \mathrm{ml}$ để xác định VK K. pneumoniae kháng với ME [6] thì mức độ đề kháng $\mathrm{KS}$ cao (gần $80 \%$ số chủng có $\mathrm{MIC} \geq 64 \mu \mathrm{g} / \mathrm{ml}$ ).

Nếu căn cứ tiêu chuẩn MIC $\geq 4 \mu \mathrm{g} / \mathrm{ml}$ để xác định kháng với COL [6] thì VK $A$. baumannii trong nghiên cứu này chưa ghi nhận trường hợp nào; còn trên $P$. aeruginosa có $6 \%$ chủng $(3 / 50)$ và $K$. pneumoniae có $10 \%$ chủng $(5 / 50)$.

Tất cả các chủng APK-CR đều kháng với CIP và cao rất nhiều lần khi so với ngưỡng đề kháng 
MIC [6].

Bên cạnh đó, Bảng 1 dưới đây còn thể hiện rõ nồng độ KS mà tại đó ức chế được 50\% (MIC50) và $90 \%$ (MIC90) số vi khuẩn, cùng với khoảng giá trị MIC từ nhỏ nhất cho đến lớn nhất (MIC range) của các loại kháng sinh $\mathrm{ME}, \mathrm{COL}, \mathrm{CIP}$ trên các chủng APK-CR.

\section{Bảng 1: MIC50, MIC90, MIC range}

\begin{tabular}{|c|c|c|c|c|}
\hline $\begin{array}{l}\text { Khá } \\
\text { ng } \\
\text { sinh }\end{array}$ & MIC & $\begin{array}{c}\text { A. } \\
\text { bauma } \\
\text { nnii } \\
n=51\end{array}$ & $\begin{array}{c}P . \\
\text { aerugi } \\
\text { nosa } \\
n=50\end{array}$ & $\begin{array}{c}\text { K. } \\
\text { pneum } \\
\text { oniae } \\
n=50\end{array}$ \\
\hline \multirow{3}{*}{ ME } & MIC50 & 32 & 256 & 32 \\
\hline & & 64 & 512 & 512 \\
\hline & & $4-128$ & $4-1024$ & $4-1024$ \\
\hline \multirow{3}{*}{ COL } & & 1 & 1 & 1 \\
\hline & MIC9 & 1 & 2 & 2 \\
\hline & & $0,5-2$ & $0,5-8$ & $0,25-8$ \\
\hline \multirow{3}{*}{ CIP } & & 64 & 32 & 64 \\
\hline & MIC90 & 128 & 128 & 128 \\
\hline & range & $8-128$ & $2-128$ & $2-128$ \\
\hline
\end{tabular}

3.2. Hiêu quả phối hợp KS. Sau khi thực hiện phối hơơp kháng sinh ME-COL, ME-CIP và tính chỉ số FIC, ta có kết quả như sau:

\begin{tabular}{|c|c|c|c|}
\hline $\begin{array}{l}\text { Kết } \\
\text { quả }\end{array}$ & $\begin{array}{c}\text { 2: Ket qua } \\
\text { baumannii }\end{array}$ & $\begin{array}{c}\text { phoi hop M } \\
\text { P. } \\
\text { aeruginosa }\end{array}$ & $\begin{array}{c}\text { K.pneumon } \\
\text { iae }\end{array}$ \\
\hline $\begin{array}{l}\text { phoí } \\
\text { hop }\end{array}$ & n (\%) & n (\%) & n (\%) \\
\hline $\begin{array}{l}\text { Hiền } \\
\text { đồng }\end{array}$ & $30(58,8)$ & $16(32)$ & $10(20)$ \\
\hline $\begin{array}{l}\text { Cộng } \\
\text { hơpp }\end{array}$ & $21(41,2)$ & $30(60)$ & $30(60)$ \\
\hline ộc lập & $0(0)$ & $3(6)$ & $6(12)$ \\
\hline $\begin{array}{l}\text { Đôi } \\
\text { háng }\end{array}$ & $0(0)$ & $0(0)$ & $0(0)$ \\
\hline
\end{tabular}

Kết quả phối hợp KS in vitro ME-COL trên APK-CR (Bảng 2) cho hiệu quả hiệp đồng và công hợp với tỷ lê lần lượt là $58,8 \%$ và $41,2 \%$, $32 \%$ và $60 \%, 20 \%$ và $60 \%$.

Bảng 3: Kêt quả phôi hợp ME-CIP

\begin{tabular}{|l|c|c|c|}
\hline $\begin{array}{c}\text { Kết quả } \\
\text { phối hợp }\end{array}$ & $\begin{array}{c}\text { A. } \\
\text { baumannii }\end{array}$ & $\begin{array}{c}\mathbf{P .} \\
\text { aeruginosa }\end{array}$ & $\begin{array}{c}\text { K. } \\
\text { pneumon } \\
\text { iae }\end{array}$ \\
\cline { 2 - 4 } & $\mathbf{n}(\%)$ & $\mathbf{n}(\%)$ & $\mathbf{n}(\%)$ \\
\hline Hiệp đồng & $17(33,3)$ & $15(30)$ & $21(42)$ \\
\hline Cộng hợp & $23(45,1)$ & $30(60)$ & $22(44)$ \\
\hline Độc lập & $9(17,7)$ & $4(8)$ & $2(4)$ \\
\hline Đối kháng & $0(0)$ & $0(0)$ & $0(0)$ \\
\hline Kết quả phối hợp KS in vitro ME-CIP trên \\
APK-CR (Bảng 3) cho hiệu quả hiệp đồng và \\
cộng hợp theo tỷ lệ lần lượt là 33,3\% và 45,1\%, \\
30\% và 60\%, 42\% và 44\%.
\end{tabular}

Bảng 4: Tý lề (\%) nhạy và trung gian của kháng sinh ME, COL, CIP đơn lè và khi

\begin{tabular}{|c|c|c|c|c|}
\hline \multirow{2}{*}{\begin{tabular}{|c|} 
Khá \\
ng \\
sinh \\
\end{tabular}} & \multirow{2}{*}{$\begin{array}{c}\text { Nhạ và trung } \\
\text { gian }\end{array}$} & \multicolumn{3}{|c|}{ A.baumP.aerugK.pneum } \\
\hline & & $\begin{array}{l}\text { annii } \\
(\%)\end{array}$ & $\begin{array}{c}\text { inosa } \\
(\%)\end{array}$ & $\begin{array}{c}\text { oniae } \\
(\%)\end{array}$ \\
\hline \multirow{3}{*}{ ME } & Đơn lẻ & 2 & 2 & 8 \\
\hline & Phối hợp COL & 29,4 & 10 & 8 \\
\hline & Phối hợp CIP & 5,9 & 8 & 16 \\
\hline \multirow{2}{*}{$\mathrm{COL}$} & Đơn lẻ & 100 & 94 & 90 \\
\hline & Phối hợp ME & 100 & 98 & 92 \\
\hline \multirow{2}{*}{ CIP } & Đơn lẻ & 0 & 0 & 0 \\
\hline & Phối hợp ME & 5,9 & 2 & 10 \\
\hline
\end{tabular}

Thông qua kiếm đinh Mann - Whitney, ta thấy sự khác biệt có ý nghĩa thống kê giữa hiệu quả của việc phối hợp kháng sinh $\mathrm{ME}-\mathrm{COL}$ và ME-CIP so với sử dung kháng sinh $M E, C O L$ hoặc CIP riêng lẻ $(p<0,05)$.

Trên A. baumannii:: Khi phối hợp ME-COL giúp tăng tỷ lệ nhạy và trung gian (Bảng 4) của ME từ $2 \%$ lên $29,4 \%$. Mức giảm của ME có ý nghĩa thống kê trên: 1 chủng $(2 \%)$ từ $R \rightarrow S, 13$ chủng $(25,5 \%)$ từ $\mathrm{R} \rightarrow \mathrm{I}, 1$ chủng $(2 \%)$ từ $\mathrm{I} \rightarrow \mathrm{S}$.

Khi phối hợp ME-CIP giúp tăng tỷ lệ nhạy và trung gian (Bảng 4) của ME tăng từ $2 \%$ lên $5,9 \%$, của CIP tăng từ $0 \%$ lên $5,9 \%$. Mức giảm MIC của ME có ý nghĩa thống kê trên: 2 chủng $(3,9 \%)$ từ $\mathrm{R} \rightarrow \mathrm{I}, 1$ chủng $(2 \%)$ từ $\mathrm{I} \rightarrow \mathrm{S}$. Mức giảm MIC của CIP có ý nghĩa thống kê trên: 1 chủng $(2 \%)$ từ $R \rightarrow S, 2$ chủng $(3,9 \%)$ từ $R \rightarrow I$.

Trên $P$. aeruginosa: Khi phối hợp ME-CO giúp tăng tỷ lê nhay và trung gian (Bảng 4) của ME từ $2 \%$ lên $10 \%$, của COL từ $94 \%$ lên $98 \%$. Mức giảm của ME có ý nghĩa thống kê trên: 1 chủng $(2 \%)$ từ $\mathrm{R} \rightarrow \mathrm{S}, 3$ chủng $(6 \%)$ từ $\mathrm{R} \rightarrow \mathrm{I}, 1$ chủng $(2 \%)$ từ $\mathrm{I} \rightarrow \mathrm{S}$. Mức giảm của $\mathrm{COL}$ có ý nghĩa thống kê trên 2 chủng (4\%) từ $R \rightarrow I$.

Khi phối hợp ME-CIP giúp tăng tỷ lê nhay và trung gian (Bảng 4) của ME tăng từ 2\% lên $8 \%$, của CIP tăng từ $0 \%$ lên $2 \%$. Mức giảm MIC của ME có ý nghĩa thống kê trên: 1 chủng (2\%) từ $\mathrm{R} \rightarrow \mathrm{S}$, 2 chủng ( $4 \%)$ từ $\mathrm{R} \rightarrow \mathrm{I}, 1$ chủng $(2 \%)$ từ $\mathrm{I} \rightarrow \mathrm{S}$. Mức giảm MIC của CIP có ý nghĩa thống kê trên 1 chủng $(2 \%)$ từ $R \rightarrow S$.

Trên $K$. pneumoniae: Khi phối hợp ME-CO giúp tăng tỷ lệ nhạy và trung gian (Bảng 4) của COL từ $90 \%$ lên $92 \%$. Mức giảm của ME có ý nghĩa thống kê trên 4 chủng (8\%) từ $\mathrm{I} \rightarrow \mathrm{S}$. Mức giảm của COL có ý nghĩa thống kê trên 2 chủng (4\%) từ $\mathrm{R} \rightarrow \mathrm{I}$.

Khi phối hợp ME-CIP giúp tăng tỷ lệ nhạy và trung gian (Bảng 4) của ME tăng từ $8 \%$ lên $16 \%$, của CIP tăng từ $0 \%$ lên $10 \%$. Mức giảm MIC của ME có ý nghĩa thống kê trên: 4 chủng $(8 \%)$ từ $\mathrm{R} \rightarrow \mathrm{I}, 4$ chủng $(8 \%)$ từ $\mathrm{I} \rightarrow \mathrm{S}$. Mức giảm MIC của CIP có ý nghĩa thống kê trên 4 chủng 
$(8 \%)$ từ $\mathrm{R} \rightarrow \mathrm{S}$ và 1 chủng (2\%) từ $\mathrm{R} \rightarrow \mathrm{I}$.

\section{KẾT LUẬN}

1. Các vi khuẩn APK-CR đều có MIC đề kháng cao với ME và CIP đơn lẻ với tỷ lệ từ 92$100 \%$; có $6 \%$ chủng $P$. aeruginosa và $10 \%$ chủng $\mathrm{K}$. pneumoniae là có MIC kháng $\mathrm{COL}$, chưa ghi nhận trường hợp nào có MIC kháng với COL ở A. baumannii.

2. Việc phối hợp ME-COL cho hiệu quả hiệp đồng và cộng hợp cao trên VK A. baumannii với tỷ lệ là $58,8 \%$ và $41,2 \%$. Còn đối với VK $P$. aeruginosa, K. pneumoniae cho hiêu quả hiêp đồng và cộng hợp thấp hơn với tỷ lệ lần lượt là $32 \%$ và $60 \%, 20 \%$ và $60 \%$. Phối hợp ME-COL thực hiện trên $A P K-C R$ cho kết quả tăng tỷ lệ nhạy cảm và trung gian từ $2-27 \%$ so với khi sứ dụng kháng sinh đơn lẻ.

3. Việc phối hợp ME-CIP trên VK K. pneumoniae cho tỷ lệ hiệp đồng và cộng hợp với tỷ lệ là $42 \%$ và $44 \%$. Còn với VK A. baumannii, P. aeruginosa có tỷ lệ hiệp đồng và cộng hợp với tỷ lệ lân lượt là $33,3 \%$ và $45,1 \%, 30 \%$ và $60 \%$. Phối hợp ME-CIP thực hiện trên APK-CR cũng cho kết quả tăng tỷ lệ nhạy cảm và trung gian từ 2-8\% so với khi sử dụng kháng sinh đơn lẻ.

\section{KIẾN NGH!}

Hiệu quả phối hợp KS trên các chủng APK-CR có khác nhau theo thời gian, khi có điều kiện, nên cho chỉ định xét nghiệm phối hợp kháng sinh in vitro trước để có quyểt định chính xác trong chọn lựa kháng sinh.

Cần có nghiên cứu đánh giá hiệu quả trên lâm sàng của việc phối hợp KS đối với cặp kháng sinh ME-COL và ME-CIP trong điều trị APK-CR.

Nên tiến hành mở rộng nghiên cứu hiệu quả phối hợp hai cặp kháng sinh ME-COL và ME-CIP trên các loại vi khuẩn khác.

\section{TÀI LIÊUU THAM KHẢO}

1. Nguyễn Ngọc Lân, Cao Minh Nga, Nguyễn Thị Thiên Kiều (2018), "Sự kháng thuốc của các vi khuẩn gây bệnh thướng gặp trong bệnh phẩm đường hô hấp dưới tại Bệnh viện Đại học Y Dược TP. HCM trong một năm (01/5/2016-30/4/2017)", Y hoc Thành phố Hî̀ Chí Minh, 22 (4), tr. 385-386.

2. Lương Hông Loan, Huỳnh Minh Tuấn (2020), "Trực khuẩn Gram âm tiết ESBL, AMPC, carbapenemase và phổ đề kháng kháng sinh tại Bệnh viện Đại học Y Dược TP. Hồ Chí Minh", Y học Tp Hồ Chí Minh, 24 (2), tr. 223-225.

3. Cao Minh Nga, Lê Thị Ânh Phúc Nhi và cộng sư (2014), "Sự đề kháng kháng sinh của vi khuẩn gẩy bệnh thưởng gặp tại Bệnh viện Đai học $Y$ Dược 6 tháng đầu năm 2011-2012-2013", Y học Thành phố Hồ Chí Minh, 18 (1), tr. 307-309.

\section{Cao Minh Nga, Nguyễn Thanh Bảo, Vũ Thị} Kim Cướng (2008), "Nhiếm khuẩn do Acinetobacter và tính kháng thuốc ", $\mathrm{Y}$ học Thành phố Hồ Chí Minh, 12 (1), tr. 188-193.

5. Agency European Medicines (2017), "Antimicrobial resistance", Retrieved from https://www.ema. europa.eu/en/humanregulatory/ overview/public-health-threats/ antimicrobial-resistance

6. Clinical and Laboratory Standards Institute (2020), Performance Standards for Antimicrobial Susceptibility Testing, M100, 30th ed, pp. 33-49, 174-177.

7. Tacconelli E, Magrini $\mathbf{N}$, et al. (2017), "Global priority list of antibiotic-resistant bacteria to guide research, discovery, and development of new antibiotics", World Health Organization, 27, pp. 318-327.

\section{BƯớC ĐẦU ĐÁNH GIÁ KẾT QUẢ ĐIỀU TRI FOLAT Ở BỆNH NHÂN SẨY THAI LIÊN TIẾP MANG GEN MTHFR ĐộT BIẾN}

\section{TÓM TẮT}

Sảy thai liên tiếp và đột biến gen MTHFR được ghi nhận có liên quan rõ rệt ở chủng tộc da vàng. Điều trị folat có thể làm giảm nồng độ homocystein trong máu cải thiện kết cục thai kỳ. Mục tiêu: của nghiên cứu đánh giá kết quả điều trị bằng folate đối với bệnh

${ }^{1}$ Trường Đại học Y Hà Nội

${ }^{2}$ Bênh viên phư sản Hà Nôi

Chịu trách nhiệm chính: Lê Thị Anh Đào

Email: leanhdao1610@gmail.com

Ngày nhận bài: 18.6.2021

Ngày phản biên khoa hoc: 11.10.2021

Ngày duyệt bài: 20.10.2021

\section{Lê Thị Anh Đào' ${ }^{1}$ Lưu Thị Anh ${ }^{2}$}

nhân mang gen đôt biến MTHFR C677T tại bẹnh viên Phụ Sản Hà Nội. Nighiên cứu mô tả cắt ngang trên 30 bênh nhân có tiền sử sẩy thai liên tiếp mang gen đôt biến MTHFR được điêuu trị $5 \mathrm{mg}$ Folat/ ngày trong 3 tháng. Kết quả $100 \%$ bênh nhân mang gen MTHFR dạng dị hợp tử CT. Nồng độ trung bình của homocystein trước và sau điều trị lần lượt là $10,3 \pm 3,47 ; 6,8 \pm 2,48 \mu \mathrm{mol} / \mathrm{l},(\mathrm{p}=0,000)$. Kết İuận: điều trị folat trong 3 tháng liên tục làm giảm nồng độ homocystein trong máu.

Tư khóa: sảy thai liên tiếp, đột biến MTHFR 677, homocystein

\section{SUMMARY}

